\title{
A Fundamental Plane for GAMA galaxies
}

\author{
Maritza A. Lara-López ${ }^{1}$, Andrew M. Hopkins ${ }^{1}$, Angel R. \\ López-Sánchez ${ }^{1,2}$, and the GAMA team \\ ${ }^{1}$ Australian Astronomical Observatory, PO Box 915, North Ryde, NSW 1670, Australia \\ email: mlopez@aao.gov.au \\ ${ }^{2}$ Department of Physics and Astronomy, Macquarie University, NSW 2109, Australia
}

\begin{abstract}
A full appreciation of the role played by gas metallicity $(Z)$, star-formation rate (SFR), and stellar mass $\left(M_{\star}\right)$ is fundamental to understanding how galaxies form and evolve. Using data from the SDSS-DR7 and the GAMA surveys we study the Fundamental Plane for star-forming galaxies. Our analysis allows us to confirm the existence of a Fundamental Plane, for which stellar mass $=f(Z, \mathrm{SFR})$ in star-forming galaxies.
\end{abstract}

Keywords. galaxies: abundances — galaxies: fundamental parameters — stars: formation galaxies: statistics

The existence of fundamental planes (FP) is a natural result of scaling relationships between important astrophysical properties. A FP was found by Lara-López et al. (2010) in a three dimensional study of the $M_{\star}$, gas metallicity, and SFR of SF galaxies using data from the SDSS-DR7. Lara-López et al. (2010) showed that the $M_{\star}-Z$, and $M_{\star}-S F R$ relationships are particular cases of a more general relationship, a FP. This combination reduces the scatter significantly compared to any other pair of correlations. Using GAMA and SDSS star forming galaxies, we performed a principal component analysis (PCA) to identify the underlying dimensionality of the three observables. We find that the first two principal components account for $86 \%$ and $12 \%$ of the variance, which indicates that $98 \%$ of our data can be explained in a 2 dimensional space (for a detailed explanation of this technique see Lara-López et al. 2012, in preparation). The FP for GAMA and SDSS galaxies can be seen in Fig. 1 . The $M_{\star}-Z, M_{\star}-S F R$, and $Z-S F R$ relationships are the projections of this $3 \mathrm{D}$ distribution. While $M_{\star}$ correlates with both SFR and metallicity (the well known $M_{\star}-Z$ and $M_{\star}-S F R$ relationships), the SFR does not strongly correlate with metallicity, which means that this relation is close to the face-on view of the $3 \mathrm{D}$ distribution (see top left panel of Fig. 1).
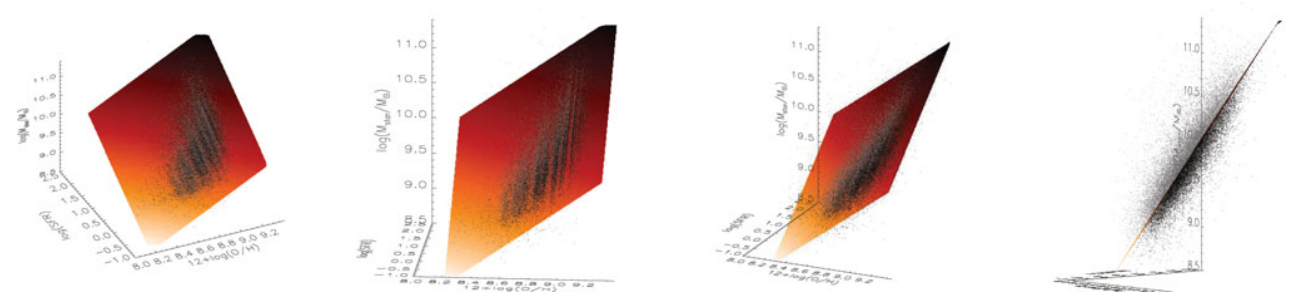

Figure 1. Projections of the 3D distribution formed by $M_{\star}, \log (\mathrm{SFR})$, and $12+\log (\mathrm{O} / \mathrm{H})$ for GAMA and SDSS galaxies. The cube is rotated clockwise from the upper-left to the bottom-right panel. Grey and black dots show galaxies above and below the FP, respectively.

\section{Reference}

Lara-López, M. A., Cepa, J., Bongiovanni, A., et al. 2010a, A\&̊A, 521, L53 